\title{
SDF-1 overexpression by mesenchymal stem cells enhances GAP-43-positive axonal growth following spinal cord injury
}

\author{
Andrew Nathaniel Stewart ${ }^{\mathrm{a}, \mathrm{b}}$, Jessica Jane Matyas ${ }^{\mathrm{a}, \mathrm{c}}$, Ryan Matthew Welchko ${ }^{\mathrm{a}, \mathrm{b}}$, \\ Alison Delanie Goldsmith ${ }^{\mathrm{a}, \mathrm{b}}$, Sarah Elizabeth Zeiler ${ }^{\mathrm{a}, \mathrm{b}}$, Ute Hochgeschwender ${ }^{\mathrm{b}, \mathrm{d}}$, \\ Ming Lu ${ }^{\mathrm{a}, \mathrm{b}, \mathrm{c}}$, Zhenhong Nan ${ }^{\mathrm{a}, \mathrm{b}, \mathrm{c}}$, Julien Rossignol ${ }^{\mathrm{a}, \mathrm{b}, \mathrm{d}}$ and Gary Leo Dunbar ${ }^{\mathrm{a}, \mathrm{b}, \mathrm{c}, \mathrm{e}, *}$ \\ ${ }^{a}$ Field Neurosciences Institute Laboratory for Restorative Neurology, Central Michigan University, \\ Mount Pleasant, MI, USA \\ ${ }^{\mathrm{b}}$ Program in Neuroscience, Central Michigan University, Mount Pleasant, MI, USA \\ ${ }^{\mathrm{c}}$ Department of Psychology, Central Michigan University, Mount Pleasant, MI, USA \\ ${ }^{\mathrm{d}}$ College of Medicine, Central Michigan University, Mount Pleasant, MI, USA \\ ${ }^{\mathrm{e}}$ Field Neurosciences Inst., 4677 Towne Centre Rd. Suite 101 Saginaw, MI, USA
}

\begin{abstract}
.
Purpose: Utilizing genetic overexpression of trophic molecules in cell populations has been a promising strategy to develop cell replacement therapies for spinal cord injury (SCI). Over-expressing the chemokine, stromal derived factor-1 (SDF-1 $\alpha$ ), which has chemotactic effects on many cells of the nervous system, offers a promising strategy to promote axonal regrowth following SCI. The purpose of this study was to explore the effects of human SDF-1 $\alpha$, when overexpressed by mesenchymal stem cells (MSCs), on axonal growth and motor behavior in a contusive rat model of SCI.

Methods: Using a transwell migration assay, the paracrine effects of MSCs, which were engineered to secrete human SDF$1 \alpha$ (SDF-1-MSCs), were assessed on cultured neural stem cells (NSCs). For in vivo analyses, the SDF-1-MSCs, unaltered MSCs, or Hanks Buffered Saline Solution (vehicle) were injected into the lesion epicenter of rats at 9-days post-SCI. Behavior was analyzed for 7-weeks post-injury, using the Basso, Beattie, and Bresnahan (BBB) scale of locomotor functions. Immunohistochemistry was performed to evaluate major histopathological outcomes, including gliosis, inflammation, white matter sparing, and cavitation. New axonal outgrowth was characterized using immunohistochemistry against the neuron specific growth-associated protein-43 (GAP-43).

Results: The results of these experiments demonstrate that the overexpression of SDF- $1 \alpha$ by MSCs can enhance the migration of NSCs in vitro. Although only modest functional improvements were observed following transplantation of SDF-1-MSCs, a significant reduction in cavitation surrounding the lesion, and an increased density of GAP-43-positive axons inside the SCI lesion/graft site were found.

Conclusion: The results from these experiments support the potential role for utilizing SDF- $1 \alpha$ as a treatment for enhancing growth and regeneration of axons after traumatic SCI.
\end{abstract}

Keywords: Spinal cord injury, stromal derived factor-1, CXCL12, GAP-43, mesenchymal stem cell

\footnotetext{
${ }^{*}$ Corresponding author: Gary L. Dunbar, Ph.D., Field Neurosciences Institute Laboratory for Restorative Neurology, Program in Neuroscience, Health Professions Building 2182, Central Michigan University, Mount Pleasant, Michigan 48859, USA. Tel.: +1 989774 3282; E-mail: dunba1g@cmich.edu.
}

\section{Research Highlights}

- MSCs were engineered to overexpress SDF- $1 \alpha$.

- SDF-1 $\alpha$ expression by MSCs enhances the migration of NSCs in vitro.

- SDF- $1 \alpha$ expression by MSCs enhanced GAP43 fibers within the lesion center. 


\section{List of Abbreviations}

$\begin{array}{ll}\text { BBB } & \begin{array}{l}\text { Basso, Beattie, and Bresnahan scale } \\ \text { for locomotor recovery } \\ \text { CSPG }\end{array} \\ \text { Chondroitin Sulfate Proteoglycan } \\ \text { CXCR4 } & \text { Chemokine Receptor-4 } \\ \text { GAP-43 } & \text { Growth Associated Protein-43 } \\ \text { GFAP } & \text { Glial Fibrillary Acetic Protein } \\ \text { GFP } & \text { Green Fluorescent Protein } \\ \text { IBA-1 } & \text { Ionized Calcium Binding } \\ & \text { Adaptor Molecule-1 } \\ \text { MSC } & \text { Mesenchymal Stem Cell } \\ \text { NSC } & \text { Neuronal Stem Cell } \\ \text { SCI } & \text { Spinal Cord Injury } \\ \text { SDF-1 } \alpha & \text { Stromal Derived Factor-1 } 1 \alpha\end{array}$

\section{Introduction}

Contusion of the spinal cord in rats produces similar neuropathological events as those that occur after spinal cord injury (SCI) in humans. Unfortunately, SCI-induced disabilities that are observed in humans often are more substantial, which can limit the ability to translate treatments from animal models of SCI to clinically meaningful outcomes (Metz et al., 2000; Somers, 2000). In laboratories across the world, intervention strategies for SCI emphasize four major mechanisms for improving outcomes, including retraining motor abilities, replacing lost tissue, regenerating damaged connections, and/or retaining tissue from necrosis during the propagation of secondary injuries that occur following trauma (Alluin et al., 2014; Taylor, Jones, Tuszynski, \& Blesch, 2006; Yasuda et al., 2011; Zendedel et al., 2012). Stem cell therapies show promise as a treatment after SCI in terms of providing trophic support, replacing lost cells, and regenerating functional neuronal architecture. The trophic support provided by mesenchymal stem cell (MSC) transplants attenuates secondary injury cascades and fills lesion cavities with a permissive substrate suitable for regrowth (Alto et al., 2009; Song, Xu, Zhang, Ma, \& Zhao, 2014). The trophic factors released from MSCs, in addition to the growth-sustaining extracellular matrix provided by the transplant, may also be capable of promoting regeneration of axons into, or beyond, the scar tissue produced by the injury (Kadoya et al., 2009). The identification of the ability for MSCs to secrete trophic factors as a mechanism to support recovery has led to the initiation of studies that utilize genetic engineering of MSCs to enhance the release of important molecules in attempts to further mitigate secondary injury cascades and promote cellular survival (Crane, Rossignol, \& Dunbar, 2014; Dey et al., 2010; Ruitenberg et al., 2005; Sasaki et al., 2009; Shang et al., 2011; Zhang et al., 2012).

The goal of the current study was to determine whether the genetic overexpression of a known chemokine protein, human stromal derived factor$1 \alpha$ (SDF-1 $\alpha$; also known as CXCL12), can improve pathological outcomes, and/or increase the growth of axons into the transplant site, compared to treatment with MSCs alone. SDF- $1 \alpha$ has been reported to function as an axonal guidance cue during development of many central nervous system (CNS) structures, including the substantia nigra, cerebellum, olfactory bulb, and corticospinal tracts, making the increased expression of SDF- $1 \alpha$ following SCI a potentially efficacious strategy for encouraging axonal growth post-transplantation (Bodea et al., 2014; Lieberam, Agalliu, Nagasawa, Ericson, \& Jessell, 2005; Toba, Tiong, Ma, \& Wray, 2008; Zhu, Matsumoto, Mikami, Nagasawa, \& Murakami, 2009). Additionally, the major receptor to SDF- $1 \alpha$, CXCR4, is expressed in almost all CNS cell types. As such, it is hypothesized that the overexpression of SDF-1 $\alpha$ will promote the survival of tissue following SCI (Li \& Ransohoff, 2008; Zendedel et al., 2016).

An in vitro portion of the present study was designed to confirm that SDF- $1 \alpha$ induces cell migration. The in vivo portion of the present study furthered the in vitro findings to assess if transplants of SDF$1 \alpha$ MSCs could stimulate axonal regrowth, mitigate SCI-induced cell pathology, and reduce functional deficits in rats that were given severe spinal cord contusions. Specifically, measures of axonal growth, gliosis, white matter integrity, inflammation, and motor functions using the Basso, Beattie, and Bresnahan scale for locomotor function (BBB; (Basso, Beattie, \& Bresnahan, 1995), were made to assess the potential therapeutic efficacy of transplanted MSCs and MSCs that were genetically engineered to overexpress SDF- $1 \alpha$.

\section{Methods}

\subsection{Engineering of MSCs that overexpress SDF- $1 \alpha$}

Cloning of the human SDF-1 $\alpha$ was originally performed by Orimo and colleague (2005), and this clone was purchased from Addgene (Cambridge, MA) 
as a stab culture containing the cDNA copy on a pBABE-puro retroviral backbone. This pBABE-puro SDF- $1 \alpha$ was a gift from Bob Weinberg (Addgene plasmid \# 12270; (Orimo et al., 2005). The SDF-1 $\alpha$ open reading frame was re-cloned from the obtained cDNA with the addition of Bgl-II restriction site and a synthetic translation initiation Kozak sequence (GCC GCCACC) on the 5' N-terminus immediately before the ATG start codon (Kozak, 1987) and the BAM-HI restriction site at the 3' C-terminus, with removal of the TAA stop codon. Similarly, a T2A peptide sequence, linked by restriction site Xhol to the reporter gene, green florescent protein (GFP), was cloned with no restriction site on the 3' end, and with the addition of BAM-HI to the 5' end. Forward cloning primers to SDF- $1 \alpha$ contained the sequence 5'-AGATCTGCCGCCACCATGAACGCCAAGGT CGTG-3', while reverse SDF-1 $\alpha$ cloning primers were designed as 5'-GGATCCCTTGTTTAAAGCT TTCTCCAGG. The T2A-GFP sequence was previously constructed in our lab and was re-cloned with forward primers 5'-GGATCCGGCAGTGGAGAG GGCAGAGGAAG-3' and reverse primers 5'-TTAC TTGTACAGCTCGTCCATGGCCGAGAG-3'. The polymerase-chain reaction (PCR) cloned gene fragments were ligated onto commercially available T-Easy cloning vectors using T4 DNA ligase (New England Bio-Labs, Ipswich, MA), transformed and grown in JM 109 strain E. coli, purified using a Qiagen Mini Prep spin kit (Qiagen Inc., Limberg, Neatherlands), and recombined through restriction digestions and subsequent ligations, to formulate the sequence of interest SDF-1 $\alpha-\mathrm{T} 2 \mathrm{~A}-\mathrm{GFP}$ onto the T-easy vector within a single open reading frame. Following sequencing, PCR and restriction digestion conformation, the sequence of interest was again recombined into the MSCV-puromycin-resistant retroviral vector, followed by transformation and purification as described above. The completed viral vector was transfected using calcium chloride into the PT67 packaging cell lines (CloneTech Laboratories Inc., Mountain View, CA) for the establishment of a continual production of a replication-incompetent virus. Viral containing supernatants were used to establish MSCs that over-express either SDF- $1 \alpha /$ GFP or just GFP alone. Once GFP expression was detected, an antibiotic selection was performed to obtain a pure population of transduced MSCs. Confirmation and quantification of SDF- $1 \alpha$ overexpression was performed using real-time PCR (RT-PCR) by comparatively analyzing SDF- $1 \alpha$ expression against MSCs alone, with mRNA templates obtained from homogenized MSC cell pellets.

\subsection{MSC and NSC culture}

MSCs were obtained from the femur bone marrow of a 3-month-old adult male Sprague Dawley rat, and expanded in Modified Eagles Medium (MEM) alpha, containing $10 \%$ fetal bovine serum (FBS), $10 \%$ horse serum (HS) and $1 \%$ penicillin and streptomycin (P/S) (Invitrogen Co., Carlsbad, CA). MSCs were passaged at approximately $70-80 \%$ confluence, using $0.05 \%$ trypsin/ethylenediaminetetraacetic acid (EDTA) and subsequent deactivation using 20\% horse serum (Invitrogen Co., Carlsbad, CA). Neural stem cells (NSCs) were isolated from whole brain extraction of rat pups at embryonic day-18 and expanded in media, consisting of $20-\mathrm{ng} / \mu \mathrm{L}$ epidermal growth factor (EGF), 20-ng/ $\mu \mathrm{L}$ basic fibroblast growth factor (bFGF), 1X B27 supplement without vitamin A, 1X N2 supplement, 1X Glutamax, in Neurobasal-A. Neurospheres reached an average size of $200-300-\mu \mathrm{m}$ in diameter and were passaged using Accutase (Sigma Aldrich CO., St, Louis, MO) to bring cells into a single cell suspension.

\subsection{Transwell migration assay}

As SDF- $1 \alpha$ is known to induce migration of NSCs both in vitro and in vivo, the chemotactic effects of SDF- $1 \alpha$ secretion by the modified MSCs were examined using a transwell migration chamber. SDF-1-MSCs or unmodified MSCs were seeded at 100,000-cells per well in a 24-transwell system (Corning Co., Corning, NY) in Neurobasal-A medium with $1 \% \mathrm{P} / \mathrm{S}$, and allowed to incubate for 12-hours post-seeding. At 12-hours post-seeding of the MSCs, an $8-\mu \mathrm{m}$ pore diameter, poly-L-lysinecoated transwell insert was used to separate 200,000 Hoechst-labeled NSCs from the lower chamber. NSCs were passaged into single cell suspension using cell dissociation buffer (Sigma Aldrich CO., St, Louis, MO) and manual pipetting to avoid digestion of sensitive receptors. Prior to seeding NSCs, Hoechst 33258 was added to the culture at a concentration of $1 / 1000-\mu \mathrm{L}$ for 30 minutes and washed 4 times by centrifugation and re-suspension in $50-\mathrm{mL}$ PBS. Following NSC passage, NSCs were suspended in Neurobasal-A and $1 \% \mathrm{P} / \mathrm{S}$ at 2,000-cells/ $\mu \mathrm{L}$, and $100-\mu \mathrm{L}$ of this cell suspension was placed within each transwell insert of SDF-1-MSCs, unmodified MSCs, and no-cell control wells $(n=4)$. At 12-hours 
post-NSC-seeding, images were taken of the lower wells and all suspended and adhered cells were collected by enzymatic dissociation, using $0.05 \%$ trypsin EDTA. Cells were pelleted and suspended in 500$\mu \mathrm{L}$ PBS and fluorescently Hoechst-labeled nuclei were counted with a hemocytometer and presented as cells $/ \mu \mathrm{L}$.

\subsection{Spinal cord injury}

Contusion injuries, using a 25-mm drop from the NYU MASCIS Model II Impactor, were performed on 3-4 month old female Sprague Dawley rats, which weighed between 250 and 350 grams. Under sterile surgical conditions, rats were anesthetized and maintained under isofluorane for the duration of the procedure. Backs of the rats were shaved and sterilized with chlorohexadine, before making a $2.5-5 \mathrm{~cm}$ incision over the $\mathrm{T} 8$ vertebrae. Fat, fascia, and muscle were retracted from the dorsal process of the $\mathrm{T} 8$ vertebra before performing a laminectomy, ensuring that the dura remained intact. Additional muscle was retracted from the $\mathrm{T} 7$ and $\mathrm{T} 9$ vertebrae to allow for stabilization of the rat via vertebral clips before dropping the 10-gram weight onto the dorsal surface of the spinal cord. A small globule of subcutaneous fat was dissected and placed over the surface of the dura to help prevent fibrosis for re-exposure, and the muscles, fascia, and skin were sutured. Rats were given bimodal analgesia consisting of $0.02 \mathrm{mg} / \mathrm{kg}$ buprenorphine (Reckitt Benkiser Pharmaceuticals, Richmond, VA), and $1.0 \mathrm{mg} / \mathrm{kg}$ meloxicam (Boehringer Ingelheim Vetmedica Inc. St. Joseph, MO) twice a day, for three days following injury. Animals were housed in pairs, except for the first three days post-injury, at which time they were housed in individual cages, and were monitored for post-operative complications. Issues arising postoperation were limited to rare occasions of urinary tract infections, which required the administration of a three-day treatment of $10 \mathrm{mg} / \mathrm{kg}$ Enrofloxacin (Baytril; Med-Vet International, Mettawa, IL). Manual bladder expressions were performed four times per-day for the first week post-injury, and twice per day there-after.

\subsection{Transplantation into the spinal cord}

Nine-days post-operation, animals were anesthetized via isofluorane, and the lesion site was re-exposed under sterile conditions. A 26-gauge Hamilton syringe, containing five microliters of either 300,000 cells (passaged between 35-43 times) and suspended in Hanks Buffered Saline Solution (HBSS), or HBSS alone, was lowered approximately $1.5 \mathrm{~mm}$ ventral to the dura at approximately a 45 degree angle, relative to the surface of the spinal cord and just left of the central vein, into the lesion epicenter. Five microliters were injected at a rate of $0.5 \mu \mathrm{l} / \mathrm{min}$, and the needle was maintained inside the spinal cord for at least three minutes post-injection. The rats were supported using vertebral clips to minimize respiratory movements during transplantation. Following transplantation, the muscles, fascia and skin were again sutured and the animals were given $1.0 \mathrm{mg} / \mathrm{kg}$ meloxicam for three days post-operation. Prior to transplantation, rats were assigned to three groups based on BBB scores obtained at one-week post-injury, to ensure that all groups had baseline scores within 0.25 standard deviations from each other.

\subsection{Behavioral analysis}

One-week prior to injury, rats were acclimated to a circular plastic open-field ( $90 \mathrm{~cm}$ diameter) from which the BBB measurements were obtained. BBB scores were taken one-day prior to injury, and once each week following SCI, for seven-weeks, prior to euthanasia. As indicated above, each rat was assigned to one of the three study groups: SDF-1MSC $(n=18)$, MSC $(n=17)$, or vehicle $(n=19)$, as well as a non-injured control group $(n=5)$. For BBB assessments, the rats were allowed to freely explore the open field for up to four minutes while two experienced raters evaluated the motor abilities. A small sub-sample of rats from each group was randomly selected to be allowed to survive for up to 15 weeks to determine the safety and long-term effects of the stem cell transplant. For this chronic-survival period, 4 rats were selected in the SDF-1-MSC-treatement group, while 5 rats were selected for the MSC-only group, and 3 rats were selected from the vehicletreated group.

\subsection{Histology}

At seven weeks post-injury, rats were euthanized via overdose of pentobarbital (Fatal Plus, Med-Vet International, Mettawa, IL) and transcardially perfused, using $4 \%$ formalin prepared from paraformaldehyde (Sigma Aldrich CO., St, Louis, MO). Following perfusion, the spinal cords were extracted and post-fixed overnight in $4 \%$ formalin, 
before being transferred to $30 \%$ sucrose (Sigma Aldrich CO., St, Louis, MO). Once the tissue was equilibrated to a $30 \%$ sucrose solution the spinal cords were cut into $2-\mathrm{cm}$ longitudinal sections and frozen in methyl butane (Sigma Aldrich CO., St, Louis, MO), prior to short-term storage at $-80^{\circ} \mathrm{C}$. Tissue was later sectioned, sagittal, at a $25-\mu \mathrm{m}$ thickness and placed into $0.1 \mathrm{M}$ phosphate-buffered in saline (PBS), and these free-floating sections were labeled using standard immunohistochemical procedures.

As the total white matter sparing around a crosssection of the lesion has been associated with the strongest correlate to behavior (Basso et al., 1995), tissue from a small random sample from each group (SDF-1-MSC, $n=4$; MSC, $n=5$; vehicle, $n=6$ ) was cut in coronal cross-sections for white matter analysis. Sections of spinal cords were cut into $1-\mathrm{cm}$ longitudinal segments, immersed in M-1 Embedding Matrix (Invitrogen Co., Carlsbad, CA), cut crosssection at $30-\mu \mathrm{m}$, and directly mounted as serial sections spaced $360-\mu \mathrm{m}$ apart.

Astrogliosis and scarring were analyzed with antibodies against glial fibrilary acetic protein $(\mathrm{Rb} x$ GFAP; 1:1000; ab7260; AbCam Co., Cambridge, UK), and chondroitin sulfate proteoglycans (Ms x CSPG; 1:2000; MAB1581; Millipore Co., Billerica, MA). Inflammation was evaluated using antibodies against ionized calcium-binding adaptor molecule 1 (Rb x IBA-1; 1:9000; 019-19741; Wako Co., Richmond, VA), and white matter sparing was characterized by histological processing with eriochrome cyanine (Sigma Aldrich CO., St, Louis, MO). Immunohistochemistry was developed with avidin/biotin complex (ABC) with horseradish peroxidase (HRP) (Vector Laboratories Inc., Burlingame, $\mathrm{CA}$ ) and diaminobenzidine (DAB) with nickel enhancement. The tissue was analyzed using optical densitometry using the NIH image analysis software, Image J. To evaluate the growth of axons inside the lesion site, immunohistochemistry labeling against growth-associated protein-43 (GAP43; Rb x GAP-43; 1:500; EP890Y; AbCam Co., Cambridge, UK) was performed using $\mathrm{ABC} / \mathrm{DAB}$, with nickel enhancement. The region inside the lesion of whole tissue sections was imaged using a stereomicroscope (Nikon Optiphop-2 containing a JAI CV-S3200 camera; Nikon Co., Tokyo, Japan) and analyzed with Image J. Fluorescent immunohistochemistry labeling of GAP-43 was done to confirm that the localization of the label was inside the lesion site.
For analysis of tissue labeled with eriochrome cyanine, GFAP, CSPG, and IBA-1, densities of regions on the longitudinally sliced tissue sections $(\sim 1 \mathrm{~cm}$ away from the lesion epicenter) were measured and used to normalize the densitometry results. Eriochrome cyanine staining on cross-sections were analyzed by tracing the spared white matter, and identifying the section containing the smallest spared volume for identification of the lesion epicenter. This lesion epicenter was used for directly comparing the total area of spared white matter at defined distances rostral and caudal to the lesion. For longitudinal sections, the image containing a 5255- $\mu \mathrm{m}$-long section of tissue surrounding the lesion site was analyzed. All images acquired were processed and analyzed using blinded volunteers. Sagittal GFAP-labeled sections were analyzed for staining densities by tracing around the lesion, excluding cavitation and tissue inside the glial scar borders, while sagittal CSPG- and IBA-1labeled sections were analyzed by tracing all tissue, excluding cavitation, and sagittal eriochrome cyanine sections were analyzed as whole tissue sections, including the lesion cavity. All data from sagittal GFAP-, CSPG-, IBA-1- and eriochrome cyaninelabeled tissue was presented as a percent optical density, standardized to regions at approximately $1-\mathrm{cm}$ from the lesion epicenter. For sagittal GAP43-labeled sections, images at the lesion epicenter were taken with a 10x objective, using standard light microscopy and the region inside the penumbra (tissue and cavitation) was traced and reported as mean optical density. For all optical density measurements, lower values suggest darker images, and more labeling/staining. To estimate total cavitation, the area of the lesion was determined by identifying the GFAP scar borders in sagittal sections, and the total area was traced, followed by tracing tissue inside the lesion and measuring the percent cavitation (see Fig. 7). Lesion boundaries were clearly visible in both GFAP and GAP-43 labeled sections which was characterized by the abrupt transition of tissue absent in stain (see Fig. 7).

For analyzing tissue histopathologies in sagittal cut sections, every twelfth tissue section (cut serially and spaced 300- $\mu \mathrm{m}$ apart) was mounted and analyzed for each animal (SDF-1-MSC, $n=7$; MSC-only, $n=7$; Vehicle-treated, $n=7$ ). Tissue sections disrupted during the sectioning, mounting or staining process were not included in analysis. For GFAP-labeling, an average of $4.73(\mathrm{SD}=1.13)$ sections-per-animal were analyzed. For CSPG- labeled sections, an average of $4.95(\mathrm{SD}=0.83)$ sections-per-animal were 
analyzed. For eriochrome-cyanine-stained sections, an average of $5.54(\mathrm{SD}=0.97)$ sections-per-animal were analyzed. For IBA-1-labeled sections, an average of 4.93 ( $\mathrm{SD}=0.84)$ sections-per-animal were analyzed. For GAP-43-labeled sections, only tissue samples containing lesion boundaries were traced, averaging $4.01(\mathrm{SD}=1.12)$ sections per-animal. For analyzing cavitation, only tissue samples containing distinct GFAP-stained boundaries were traced, averaging $4.10(\mathrm{SD}=1.10)$ sections per-animal.

\subsection{Statistical analysis}

For BBB measurements, the non-parametric Kruskall-Wallis analysis of variance (ANOVA) was utilized to compare data obtained from non-injured, SDF-1-MSC, MSC-only, and vehicle treatment groups for 7-weeks post-injury. For the sub-group of rats allowed to survive for 15-weeks post-injury, the Friedman's non-parametric repeated measures ANOVA was used to determine whether changes occurred within each group from 7-weeks to 15weeks post-injury. A one-way ANOVA was used for comparing SDF-1-MSC, MSC-only, and vehicle treatment groups on all other measures, using Statistical Package for the Social Sciences version 23 (SPSS). When appropriate, Fisher's Protected Least Significant Difference (PLSD) test was used for post hoc analysis of all parametric data. To determine whether meaningful relationships may exist between outcome measures, one-tailed Pearson correlations were performed between BBB scores at 7-weeks post-injury, measures of cavitation, as well as densities in GAP-43, GFAP, CSPGs, and eriochrome cyanine staining.

\section{Results}

\subsection{Cloning and construction of $S D F-1 \alpha-T 2 A-G F P$ onto MSCV-puro}

Cloning of SDF- $1 \alpha$, T2A-GFP, and recombination onto the MSCV vector were performed successfully, without mutation, as indicated by gene sequencing. Restriction digestion and PCR conformation was used to evaluate the size and orientation of the completed plasmid (Fig. 1 A-B). PT67 cells were successfully transfected before selection in puromycin to obtain a pure population of virus-producing cells.

\subsection{Transduction and expression of $S D F-1 \alpha$ in MSCs}

Viral supernatants of GFP- and SDF- $1 \alpha$-GFP producing PT67 cells were obtained after 24-hours in culture and used to infect MSCs. One day later, GFP expression in both cell lines could be detected under fluorescent microscopy (Fig. 1C). Again, the population of cells was purified with puromycin,
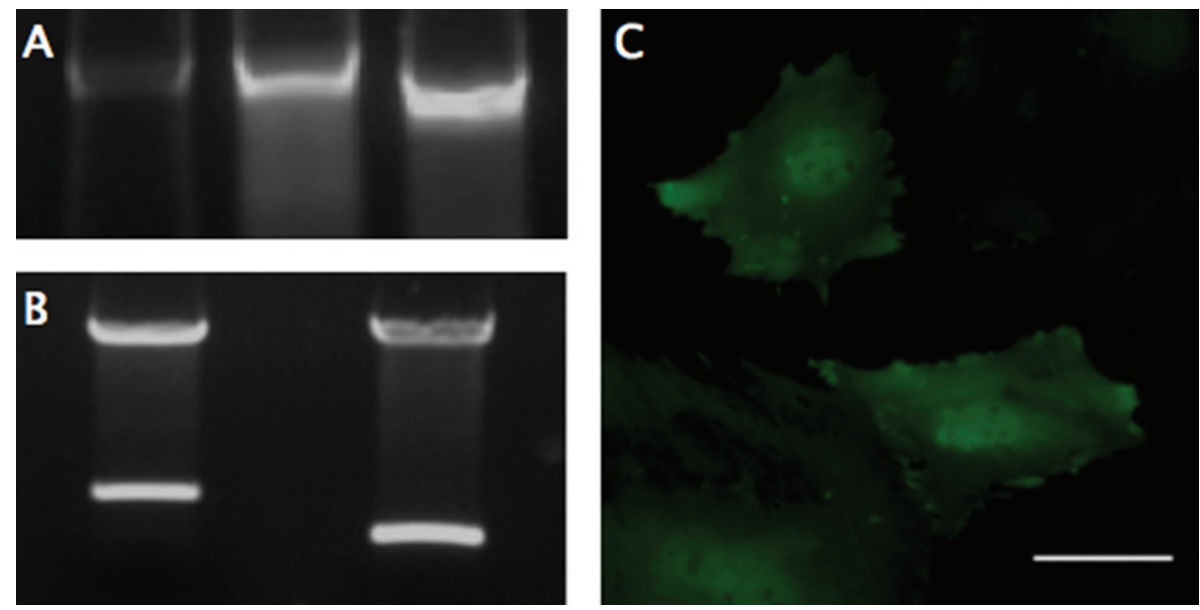

Fig. 1. PCR, Digestion, and Fluorescent Expression. (A) Genomic DNA from modified MSCs was isolated and examined with PCR using primers designed to flank the multiple cloning site, to analyze the insertion of the SDF-1-T2A-GFP sequence (Left), using the plasmid DNA (Middle) and GFP only plasmid (Right) as size controls. The genomic DNA revealed size-matched PCR products to the plasmid and a 300 larger base-pair fragment, compared to the GFP control. (B) Restriction digestion was performed on the completed MSCV-puro retroviral vector with the SDF-1-T2A-GFP sequence (Left), or just GFP sequence (Right) inserted into the multiple cloning site between $\mathrm{Bgl-II}$ and EcoRI restriction sites. (C) GFP was used as a reporter gene to confirm transcription and translation of the engineered sequence. GFP expression was confirmed using fluorescent microscopy with a FITC filter. Scale bar represents $50-\mu \mathrm{m}$. 
and the culture was allowed to recover. DNA and mRNA were obtained for analyzing the insertion and expression of the transgene with PCR, which demonstrated successful integration into the cells (Fig. 1A) and RT-PCR demonstrating a significant $(\mathrm{F}(2,3)=12.99, p<0.05) 400$ fold increase (Mean difference $=414.83, \mathrm{SD}=135.75)$ in SDF- $1 \alpha$ expression compared to non-transfected MSCs using the

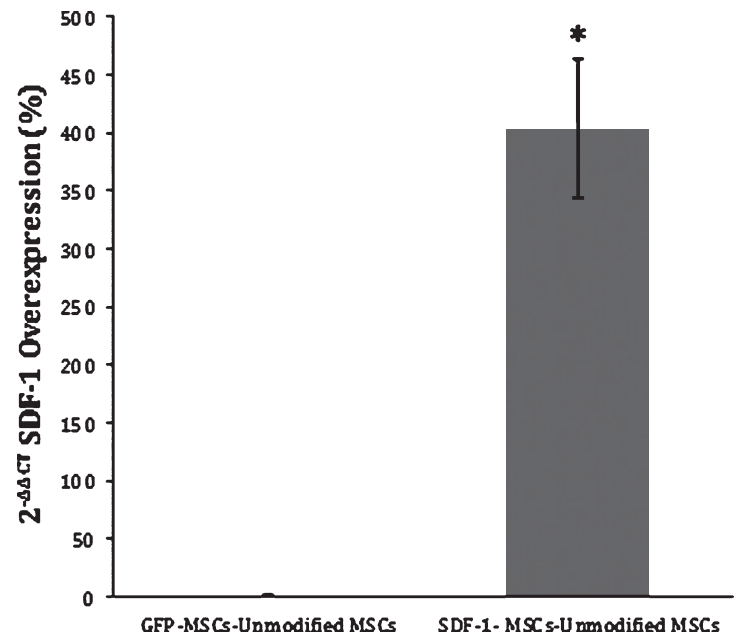

Fig. 2. SDF-1 RT-PCR Expression. The 2- ${ }^{\Delta \Delta \mathrm{Ct}}$ method was applied to the results from RT-PCR of SDF-1-MSC cDNA and MSCs that express GFP-alone (GFP-MSC) cDNA, comparing the relative SDF-1 mRNA expression to unmodified MSCs. Results demonstrate a 400-fold increase in gene expression in SDF-1MSCs compared to unmodified or MSCs that express GFP-alone. *Significance differences found at $(p<0.05)$ between SDF-1-MSC and MSC $\Delta \Delta C$ T values, relative to unmodified MSCs.
$2^{-\Delta \Delta \mathrm{Ct}}$ method $(p<0.05 ; n=2)$ (Fig. 2). A $2^{-\Delta \Delta \mathrm{Ct}}$ value of 8.856 between SDF- $1 \alpha$ expressing MSCs $(\mathrm{SDF}-1 \alpha \mathrm{Ct}=20.65$, GAP-DH $\mathrm{Ct}=19.27)$ and nontransfected MSCs (SDF-1 $\alpha \mathrm{Ct}=27.06, \mathrm{GAP}-\mathrm{DH}$ $\mathrm{Ct}=17.05$ ) was obtained, confirming transcription of the virus. A $2^{-\Delta \Delta \mathrm{Ct}}$ value of -0.14 between GFP transfected (SDF-1 $\alpha \mathrm{Ct}=27.79$, GAP-DH $\mathrm{Ct}=17.05)$ and non-transfected MSCs demonstrated that transfection, alone, does not affect the expression of SDF- $1 \alpha$.

\subsection{Function of $S D F-1 \alpha$ overexpression by MSCs in vitro}

The preservation of function by this cloned SDF-1 $\alpha$ was confirmed using the transwell chemotaxis assay. Results from this migration experiment demonstrated significant differences between groups, using a one-way ANOVA $(\mathrm{F}(2,9)=52.36, p<0.001)$, with more NSCs migrating into the lower chamber of SDF-1-MSC-wells $(\mathrm{M}=246.25, \mathrm{SD}=29.18)$ compared to both the MSCs $(\mathrm{M}=98.75, \mathrm{SD}=3.14$; $p<0.001)$ and no-MSC control wells $(\mathrm{M}=2.50$, $\mathrm{SD}=1.44 ; p<0.001)$, while MSC-alone wells also contained significantly more NSCs than no-MSC control wells $(p<0.005$; Fig. 3$)$.

\subsection{Behavior}

Behavioral analysis, using Kruskal-Wallis nonparametric analysis of variance, revealed significant
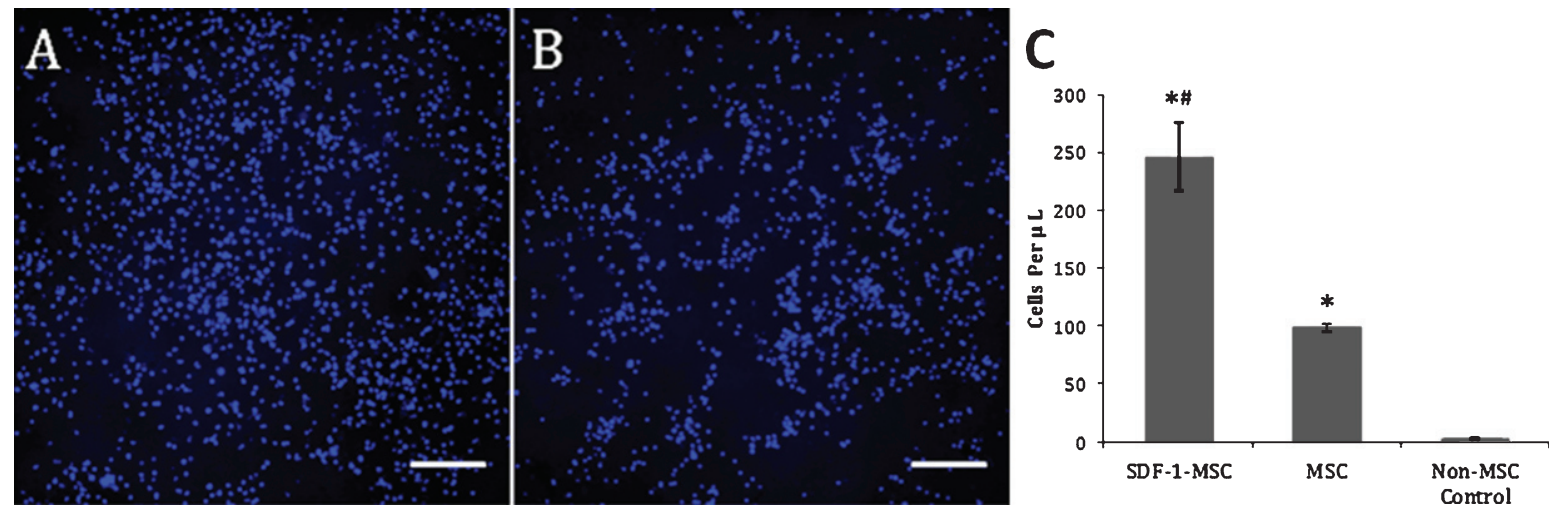

Fig. 3. Transwell Migration Assay. Representative images acquired from the center of the wells following the transwell migration assay revealed significantly greater Hoechst-labeled nuclei in SDF-1-MSC wells (A), compared to MSC-only wells $(p<0.001$; B) and non-MSC control wells $(p<0.001)$, while MSC-only wells contained significantly more Hoechst-labeled cells than in non-MSC control wells $(p<0.05)$. At 12 hours post-seeding with Hoechst pre-labeled NSCs, all cells in the bottom chamber were collected and re-suspended in 500- $\mu$ L of PBS, and a $10-\mu \mathrm{L}$ representative sample was collected and manually counted using a hemocytometer. Only Hoechst-labeled nuclei were counted, which revealed significant differences between all wells $(p<0.001 ; \mathrm{C})$. Image of non-MSC control wells is not shown due to the absence of cells in the lower chambers. Scale bars represent $200-\mu \mathrm{m} .{ }^{*} p<0.01$, compared to non-MSC control wells. ${ }^{\#} p<0.05$, relative to MSC wells. 
between-group differences at all time points $(p<0.005)$, however, as determined following individual Mann-Whitney $U$ tests, differences only existed between non-injured controls and other treatment groups $(p<0.005)$. At 7 -weeks post-injury, a trend indicating improved function of rats receiving transplants of SDF-1-MSC was observed when compared to vehicle-treated rats $(\mathrm{U}=108.50, p=0.054)$. Further analysis, using a sub-group of rats with less severe injuries, and for which rats with BBB scores less than 3 at the time of transplantation were omitted from analysis, revealed a significant treatment effect at 7-weeks post-transplantation between the SDF-1MSC- and vehicle-treated rats $(\mathrm{U}=52.50, p<0.037)$ (Fig. 4). Analyses of the rats that were allowed to survive for 15-weeks post-injury were analyzed biweekly for changes in BBB scores from 7-weeks to 15-weeks using the Friedman's test, and showed no further improvements beyond 7-weeks post-injury.

\subsection{Histology}

No between-group differences were found for optical densitometry measurements on longitudinal sections for the GFAP-labeled sections $(\mathrm{F}(2,17)=$ $0.268, p=0.768$; Fig. 5A-C), CSPG-labeled sections $(\mathrm{F}(2,17)=0.493, p=0.619$; Fig. 5D-F $)$, eriochromecyanine-stained sections $(\mathrm{F}(2,17)=0.105, p=0.901$; Fig. 5G-I), or IBA-1-labeled sections $(\mathrm{F}(2,17)=$ 1.006, $p=0.386$; Fig. 5J-L). For analyzing tissue histopathologies, every twelfth tissue section (cut serially and spaced $300-\mu \mathrm{m}$ apart) from each animal was mounted and analyzed.

Regions immediately surrounding the lesion epicenter were filled with dense, CSPG-rich tissue, with hollow, fluid-filled, cavitation existing around the scar. To measure the extent of cavitation surrounding the lesion area, the total area of cavitation was determined by tracing around regions of compromised tissue in sagittal sliced, GFAP-labeled sections, followed by tracing cavitation on image $\mathrm{J}$ and computing the percent of the injured area that was filled with tissue (Fig. 6). Tissue samples containing distinct GFAP-stained boundaries were traced. A one-way ANOVA failed to detect differences in the total lesion area $(\mathrm{F}(2,16)=0.433, p=0.656)$. However, between-group differences on the percent of lesion area filled with cavitation $(\mathrm{F}(2,16)=3.706, p<0.05)$ were found (Fig. 6E). Fisher's PLSD post-hoc analyses indicated that SDF-1-MSC-treated rats had less cavitation within the lesion area than did vehicletreated rats, but no differences were found between
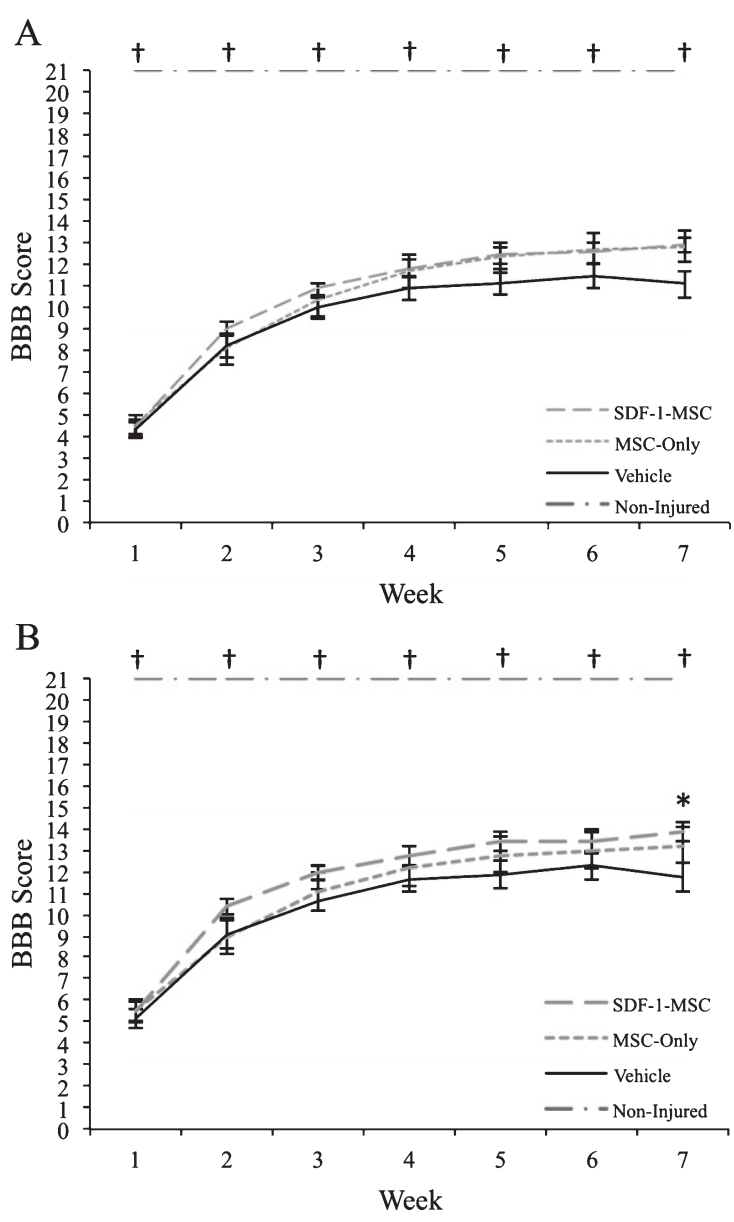

Fig. 4. Behavioral Results. Prior to transplantation at 9-days postinjury, rats were distributed into groups to ensure an equal baseline mean (BBB scores ranged from 1-9). (A) When analyzing rats with the full range of baseline scores, transplantations of SDF-1MSCs or MSCs-alone did not improve motor performance over the 7-weeks post-injury assessment period. (B) When rats with more severe injuries were removed from analysis (BBB scores ranged from 3-9), significant improvements were found with SDF1 -MSC treated rats. ${ }^{*} p<0.05$ compared with vehicle-treated rats. ${ }^{\dagger} p<0.005$ compared with all other groups.

MSC-only and vehicle-treated rats, or between SDF1-MSC and MSC-only-treated rats (Fig. 6E).

A one-way ANOVA revealed between group differences in the total area of white matter sparing at $1080-\mu \mathrm{m}$ caudal to the lesion epicenter $(\mathrm{F}(2,14)=4.399, p<0.05$; Figs. 7 and 8$)$, in crosssectional analysis of eriochrome-cyanine labeled sections. Fisher's PLSD post-hoc analysis indicated that MSC-only-treated rats $(\mathrm{M}=1148.58, \mathrm{SD}=587.61)$ had greater white matter areas compared to vehicletreated rats $(\mathrm{M}=787.49, \mathrm{SD}=641.015 ; p<0.05)$. No differences in white matter sparing were found at any of the other measured locations. 

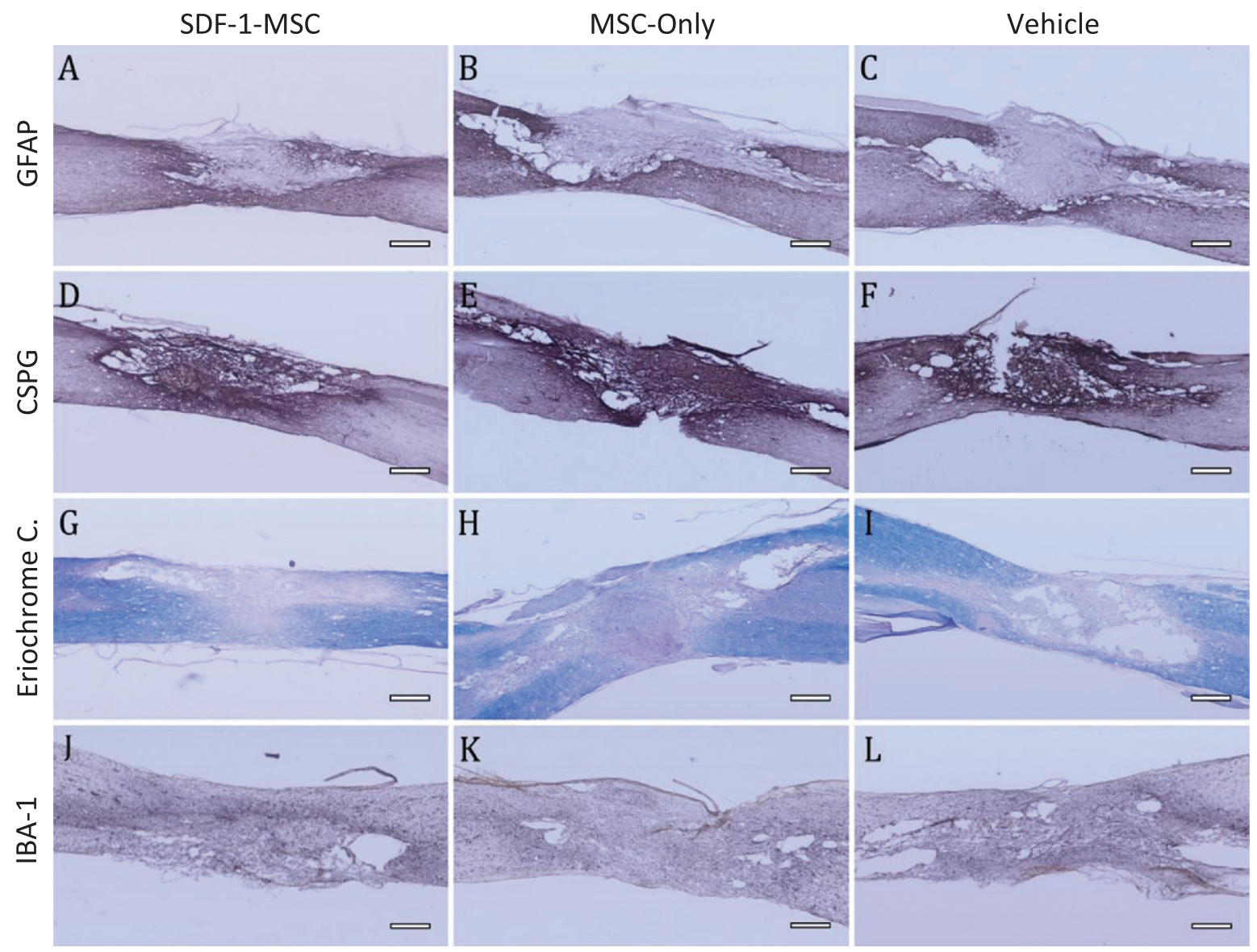

Fig. 5. Immunohistochemistry GFAP, CSPG, Eriochrome Cyanine, IBA-1. (A-I) Immunohistology was performed to analyze major histopathological outcomes for astrocytes as measured by GFAP (A-C), for proteoglycans as measured by CSPG (D-F), for white matter pathology as measured by eriochrome cyanine staining (G-I), and for inflammation as measured by IBA-1 (J-L). No significant differences could be detected between SDF-1-MSC (A,D,G,J), MSC-only (B,E,H,K), or vehicle (C,F,I,L) groups. Areas of the lesions can be identified by clear boundaries in CSPG and GFAP labeling. Tissue within the lesion area is void of immunological labeling for GFAP, but dense in CSPG labeling. Scale bars represent $500-\mu \mathrm{m}$.

To characterize the effects of SDF-1-MSCs on the growth response of axons, GAP-43 was immunologically labeled with ABC/DAB tags, followed by imaging the lesion centers with a $10 x$ objective (Fig. 9). Only tissue samples containing lesion boundaries were traced. Tissue existing within the lesion center was identified and traced using Image $\mathbf{J}$ software and the optical densities were analyzed using a one-way ANOVA, revealing significant betweengroup differences $(\mathrm{F}(2,17)=5.428, p<0.05)$. The Fisher's PLSD post-hoc analysis indicated that SDF1-MSC-treated rats had greater GAP-43 densities than did vehicle controls $(\mathrm{M}=201.49, \mathrm{SD}=16.66$; $p<0.005$ ) (Fig. 10), but were not significantly different than MSC-only rats.

\subsection{Correlation of outcome measures}

Significant negative correlations were found between end-point BBB scores and GAP-43-labeling densities $(\mathrm{R}=-0.420, p<0.05)$ for all rats in the first analysis, but not for rats with the less significant injuries $(\mathrm{R}=-0.287, p=0.141)$. This analysis suggests that animals with higher BBB scores also contained more GAP-43 labeled fibers within the lesion. Significant negative correlations also existed between densities of eriochrome cyanine staining and GFAP- $(\mathrm{R}=-0.609 ; \mathrm{R}=-0.615 ; p<0.05)$, and CSPG-labeling $(\mathrm{R}=-0.469 ; \mathrm{R}=-0.473 ; p<0.05)$ for all rats and from the sub-group of rats were less deficits, respectively. This suggests that less myelin 

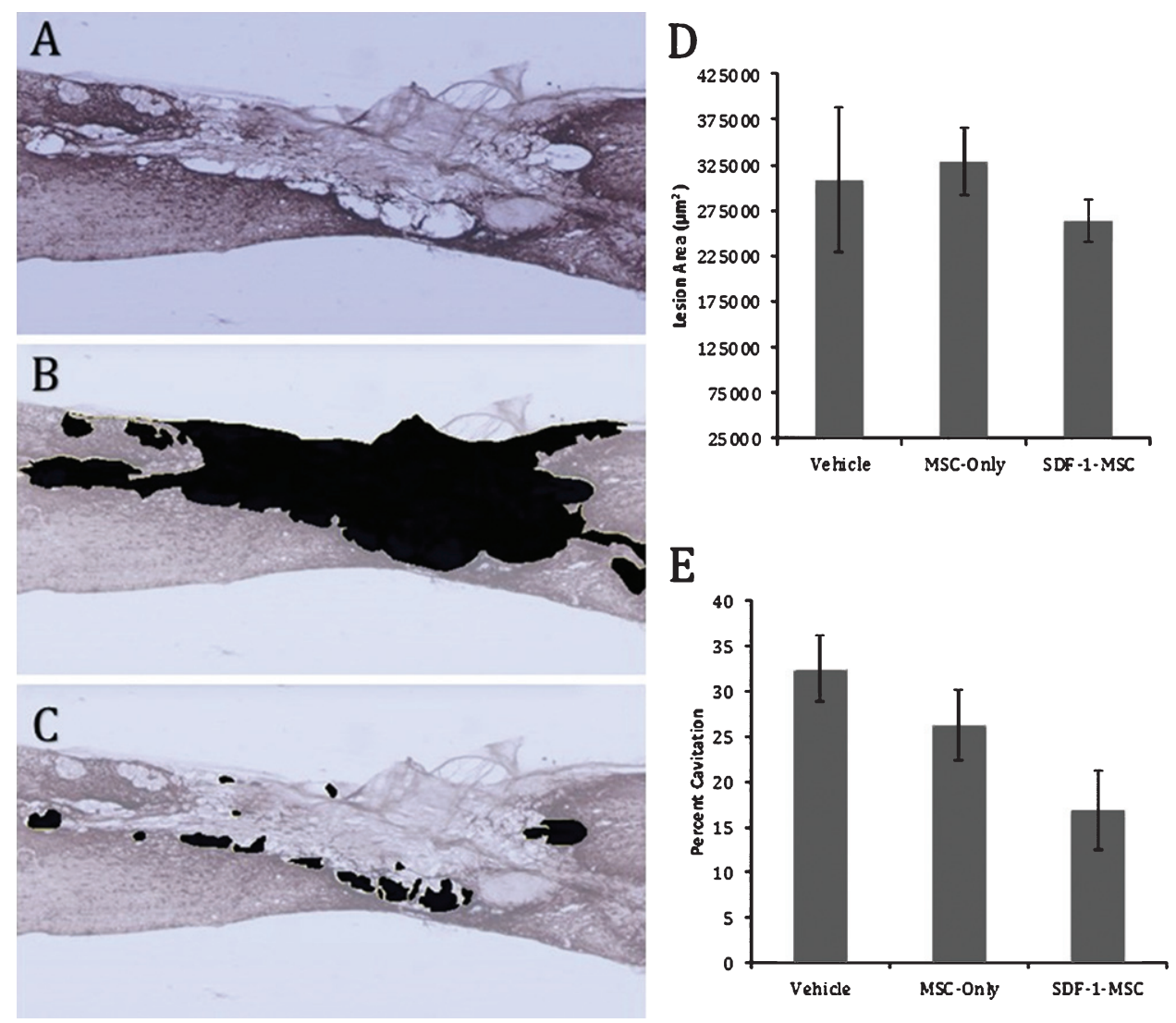

Fig. 6. Cavitation. Immunohistochemical labeling against GFAP reveals distinct borders between healthy, and scared lesion tissue (A). Tissue existing within the compromised area was traced (B,D), followed by tracing the area of cavitation (C). A one-way ANOVA was used to compare total lesion area (D), as well as the percent cavitation (E). No differences were found in the total lesion area, while differences were found between groups for percent cavitation $(p<0.05)$, with Fisher's PLSD post-hoc analysis revealing significant differences between SDF-1-MSC-treated rats and the vehicle-treated groups $(p<0.05) .{ }^{*} p<0.05$ compared with vehicle-treated rats.

surrounding the lesion correlates to more labeling with GFAP and CSPGs. Significant positive correlations were found for rats from both analyses between GAP-43 labeling and cavitation $(\mathrm{R}=0.554$; $\mathrm{R}=0.723 ; p<0.01$ ), which suggest that a greater amount of cavitation may result in less GAP-43 labeling within the lesion. Significant correlations were not found between functional outcomes and the amount of cavitation for analyses with either all rats or the sub-group with less severe deficits.

\section{Discussion}

The major findings of this study include: (1) a preserved chemotactic function of SDF- $1 \alpha$, when linked to the T2A-GFP sequence; (2) an increase in chemotactic response of NSCs towards SDF1-MSCs, compared to MSCs alone in vitro; (3) enhanced labeling of GAP-43 immunological activity within the lesion/transplant location for SDF-1MSC-treated animals; (4) significant improvements in BBB functional scores for rats that received SDF1-MSCs; and (5) reduced percent cavitation in the spinal cord of SDF-1-MSC-treated rats, without a significant inflammatory response, compared to vehicle controls. Collectively, these findings suggest that the over-expression of SDF-1 by MSCs may potentiate the regenerative effects of MSCs on axons when utilized in the appropriate circumstances.

In terms of preserved chemotactic function by SDF- $1 \alpha$, the addition of 22 amino acids onto the C' Terminal end of SDF- $1 \alpha$ from the fusion with the T2A peptide did not interfere with its chemotactic effects on neuronal stem cells. To evaluate this, we performed the in vitro chemotaxis assay, which is the best-known action that SDF- $1 \alpha$ exerts on NSCs (Chalasani, Sabelko, Sunshine, Littman, \& Raper, 2003; 


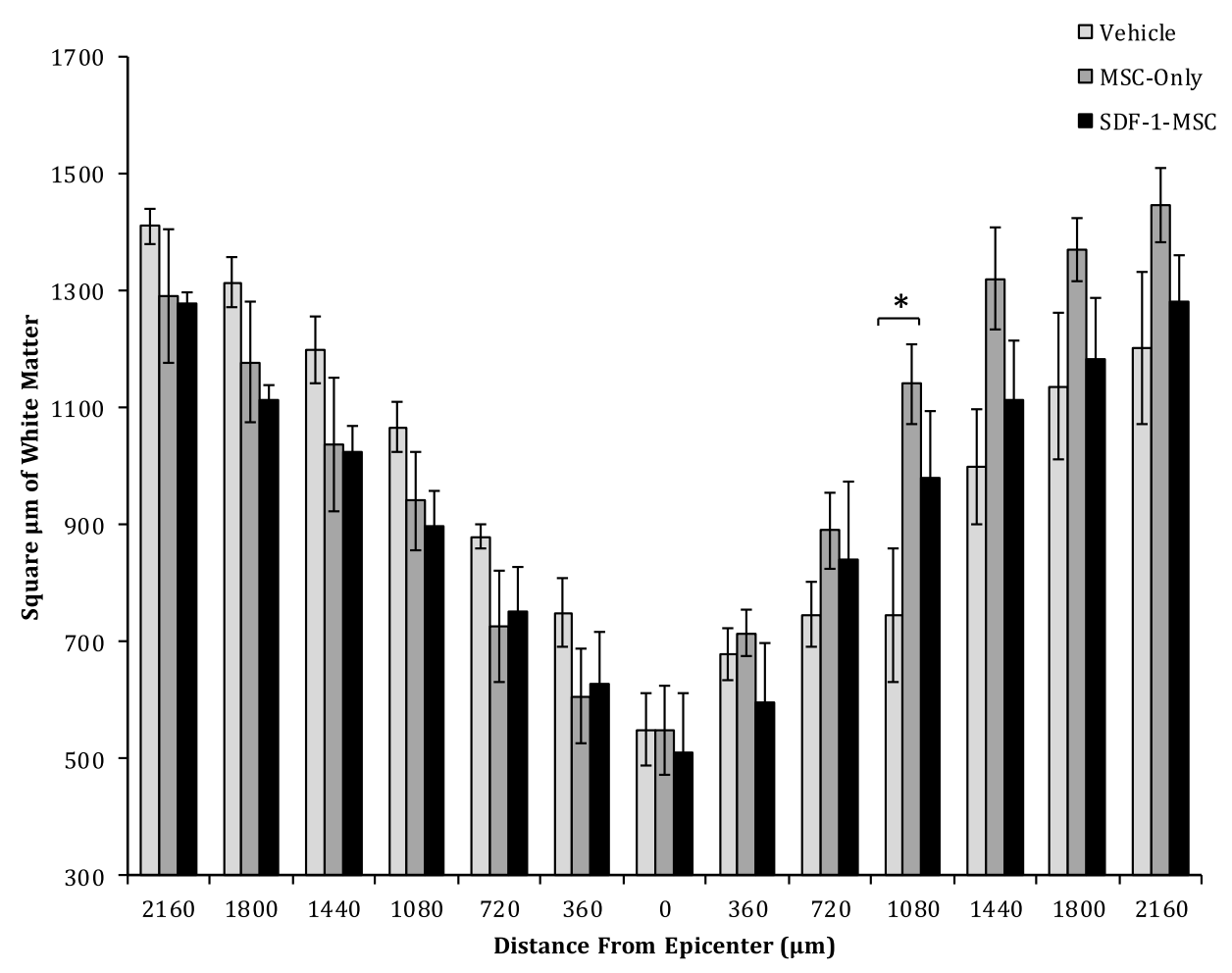

Fig. 7. White Matter Sparing. One-way ANOVAs revealed differences in the area of spared white matter at 1080- $\mu \mathrm{m}$ from the lesion epicenter between MSC-only-, and vehicle-treated rats. No significant differences were found at any other location. Spinal cords were cut serially in cross sections, spaced 360- $\mu \mathrm{m}$ apart, and analyzed for white matter surrounding the lesion. Sections containing the least spared white matter were considered the epicenter, and distances up to $2,160-\mu \mathrm{m}$ rostral and caudal to the epicenter were analyzed for each animal.

Dziembowska et al., 2005; Lieberam et al., 2005; Xue et al., 2014). The data from this study suggests that this cloned SDF- $1 \alpha$ not only maintains chemotactic functions, but also can exert greater migratory effects on NSCs in vitro, when secreted at levels exceeding those from MSCs alone. Importantly, the N' terminus of SDF- $1 \alpha$ was cloned without mutation, as manipulation of this region of the protein has been shown to be more sensitive to disrupting the chemotactic properties of these proteins (Filippo et al., 2013).

Results from this study indicate that the mean BBB scores were improved in the rats that received the transplantation of SDF-1-MSCs. Unlike previously published studies, we did not observe significance improvements in rats receiving transplants of unaltered MSCs (Oliveri, Bello, \& Biering-Sørensen, 2014). The discrepancy may be due to differences in MSC characteristics, culture conditions, and the number of passages prior to transplantation (Rossignol et al., 2015). In this study, high-passage MSCs were used, as they have been shown to produce greater treatment effects than low-passaged cells in rodent models of neurodegenerative diseases (Rossignol et al., 2015). This relationship, however, may not be consistent across different neurological pathologies, and/or species (Li et al., 2012).

Although the BBB scores were only marginally improved during our initial analysis, by removing the more severely injured animals we observed significant improvements in function for the rats receiving SDF-1-MSCs. Following SCI, functional deficits reside on a spectrum of severities. However, more severe injuries to the spinal cord may represent a much more complicated pathology compared to less severe injuries. More severe SCI will produce a greater loss of axons that span the lesion site, and will therefore rely more on mechanisms of therapy that encourage axon regeneration for restoring function. This contrasts to less severe injuries, which may be able to derive greater benefits from mechanisms of neuro-protection, re-meylination, and functional plasticity. The more axons that are damaged at the lesion site during an SCI, the less potential there is for re-myelination to occur, or for neuroprotection to exert benefits, as well as less circuitry available to retrain and be used for compensatory motor 


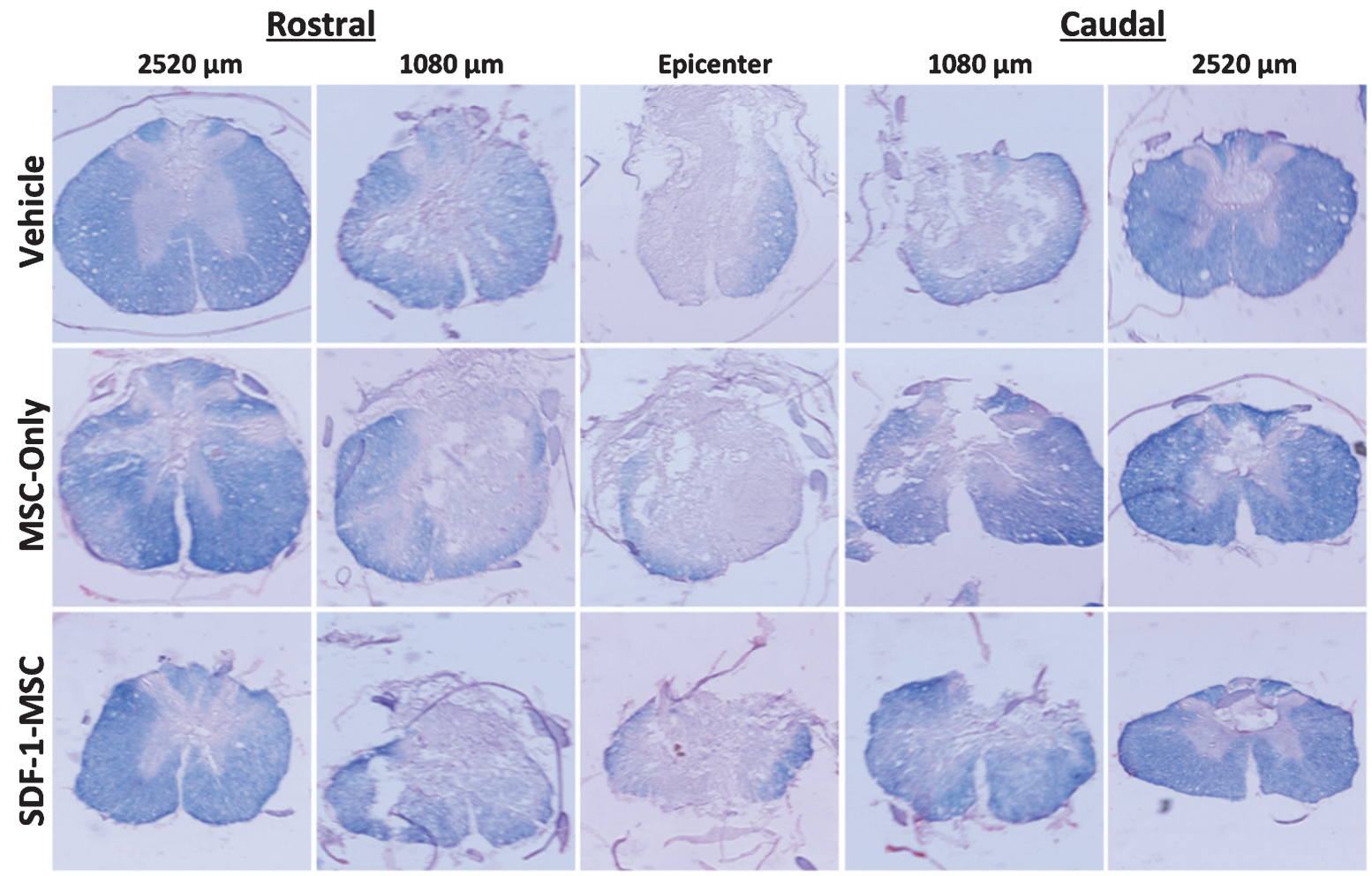

Fig. 8. Eriochrome Cyanine Cross-Section. White matter sparing was analyzed using eriochrome cyanine staining, revealing graded myelin loss surrounding the lesion epicenter. Spinal cord cross sections were cut serially, spaced $360-\mu \mathrm{m}$ apart in the rostral-caudal direction, and directly mounted. Sections were analyzed by tracing dense blue boundaries to evaluate spared white matter and compared at each representative distance from the lesion epicenter.

behaviors. The implications of this are that when modeling SCI, the variability in the severity of the lesion must be considered in the design, and limiting the range of functional abilities prior to transplantation can be a mechanism to improve predictability within the model. The results from this study demonstrated that the less-severely injured sub-population demonstrated a greater treatment effect compared to the analysis that included severely injured rats. This supports the need for future studies to analyze the relationship between the treatment potential of stem cell transplants, and the severity of the SCI.

In this study, no treatment effect was observed with the transplantations of MSCs-alone, however the overexpression of SDF- $1 \alpha$ from transplanted MSCs may improve behavioral outcomes beyond what MSCs-alone can elicit. Additionally, overexpressing SDF-1 from MSCs in this study reduced cavitation and increased GAP-43 expression within the lesionsite following SCI. This suggests that overexpressing chemokines, such as SDF- $1 \alpha$, from transplanted cells can influence histological outcomes in a manner that may provide a more permissive environment for axon regeneration, without negatively impacting function.

Transplantation of stem cells into the lesion cavity, post-SCI, has long been demonstrated to promote the growth of axons into the graft (Kumagai et al., 2013; Lu, Jones, \& Tuszynski, 2005; Neuhuber, Timothy Himes, Shumsky, Gallo, \& Fischer, 2005). The present study demonstrated that overexpressing SDF- $1 \alpha$ in MSCs can enhance the existence of GAP-43-positive axons inside the area of the lesion at a higher level than MSCs alone can provide. The overexpression of SDF- $1 \alpha$ significantly reduced cavitation surrounding the scar, and this reduction in cystic cavitation may have contributed to the overall greater ability for axons to grow inside the graft. This is suggested by the significant negative correlation found in this study between GAP-43 labeling and cavitation. This finding suggests the importance of providing an adherent substrate and extracellular matrix (ECM), as well as the secretion of attractive molecules for growing axons to follow during growth and regeneration. Such an effect has previously been demonstrated following transplantation of 


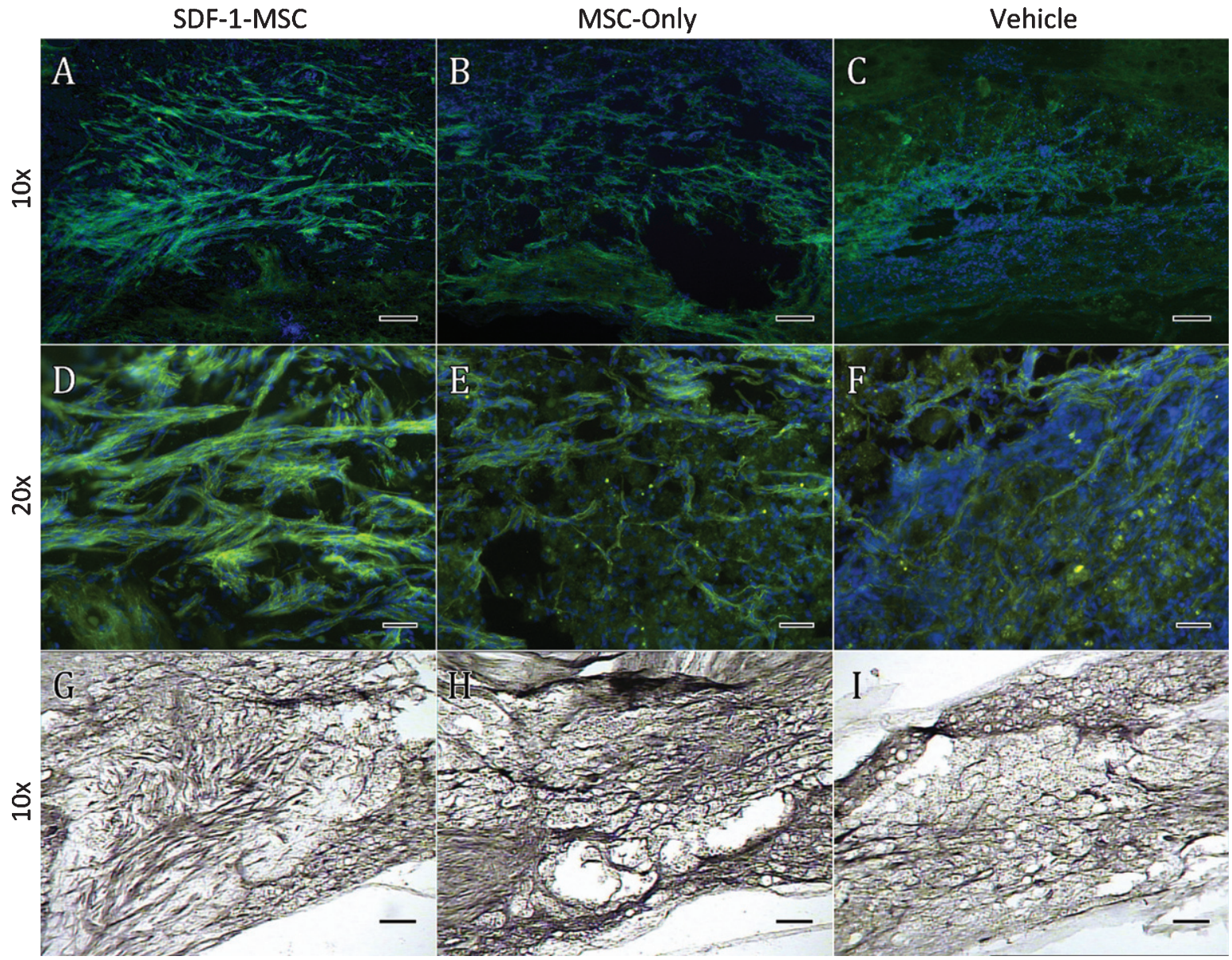

Fig. 9. Immunohistochemistry GAP-43. GAP-43 immunological labeling was found to be significantly increased within the lesion cores for SDF-1-MSC-treated (A,D,G), but not in MSC-only rats (B,E,H) compared to vehicle-treated controls (C,F,I). Data was quantified using DAB labeling (G-I) by tracing lesion cores and obtaining optical densitometry measurements using Image J. Fluorescent microscopy was utilized for imaging to confirm the specificity and localization of labeling (A-F). A 10x objective (A-C, G-I) revealed GAP-43 labeling predominately within the lesion center, while images taken with 20x objectives (D-F) revealed individual fibers or fiber bundles. Scale bars represent $100-\mu \mathrm{m}(\mathrm{A}-\mathrm{C}, \mathrm{G}-\mathrm{I})$ and $50-\mu \mathrm{m}(\mathrm{D}-\mathrm{E})$.

MSCs (Kumagai et al., 2013; Lu et al., 2005) and, as shown in this study, axonal growth can be enhanced through modifying the secretome by overexpressing chemo-attractants, such as SDF- $1 \alpha$. Due to the role of GAP-43 in the growth cone of developing or regenerating axons, it may also be likely that the effects observed in this study point towards a longer and more sustained growth response, due to the presence of MSCs that overexpress SDF- $1 \alpha$.

Although a significant correlation was found between end-point BBB scores and GAP-43 labeling, it is unlikely that the fibers found within the lesion site are contributing towards any functional improvements. Rather, this correlation likely demonstrates that a greater plasticity-like response throughout the spinal cord may lead to greater functional abilities.
Additionally, this correlation between GAP-43 labeling and BBB scores supports a mechanism by which transplanting SDF-1-MSCs may enhance functional abilities. However, what remains to be determined is whether the axon fibers existing within the graft are regenerated, spared, or are sprouting, as well as the origin of the fibers. Without demonstrating a complete transection of a spinal tract, it is impossible to conclude that fibers are regenerating, as opposed to being spared or undergoing collateral sprouting (Tuszynski \& Steward, 2012). However, as GAP-43 is found predominately within growth-stimulated axons, it is unlikely that the effects observed in this study are limited to that of spared fibers, but rather supports the notion of new growth, either from previously axotomized 


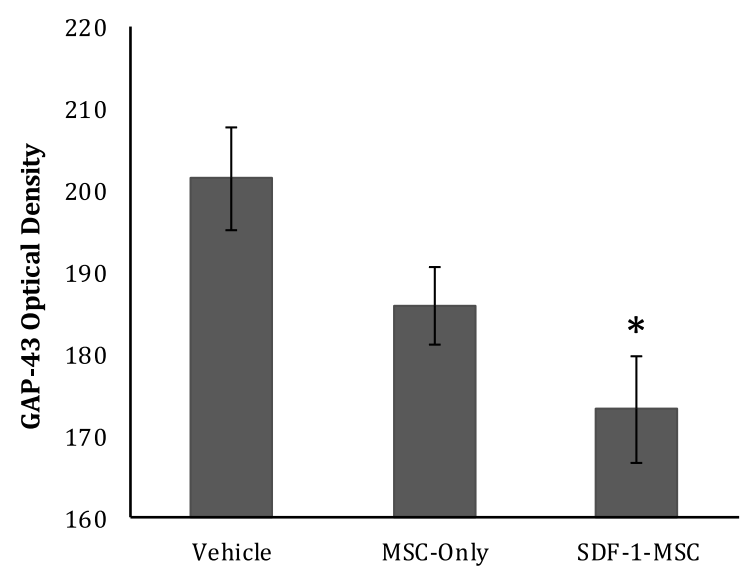

Fig. 10. GAP-43 Optical Density. GAP-43 immunological labeling was done using $\mathrm{ABC} / \mathrm{DAB}$, followed by tracing the lesion centers and evaluating optical densities. Brighter images reveal less labeling, demonstrating significantly greater GAP-43 densities in SDF-1-MSC-treated rats compared to vehicle controls $(p<0.005)$, but not between MSC-only and vehicle controls, or SDF-1-MSC and MSC-only rats. ${ }^{*} p<0.05$, relative to vehicle-treated rats.

axons, or collateral sprouting from surviving axons (Curtis, Green, Lindsay, \& Wilkin, 1993; Hoffman, 1989; Li, Kling-Petersen, \& Dahlström, 1993). Morphological assessment of GAP-43 fibers suggests that the fibers inside the damaged area displayed non-linear and sporadic directionality, which is characteristic of new process growth, further supporting the idea that these fibers are not just spared (Paul Lu, Yang, Jones, Filbin, \& Tuszynski, 2004; Tuszynski \& Steward, 2012).

Analyses with fluorescent microscopy revealed that the GAP-43 fibers were growing in bundles and almost exclusively followed Hoechst-labeled nuclei within the area of the lesion. In cell transplant groups, GAP-43 fiber bundles were not often found in regions of healthy tissue surrounding the lesion. This finding suggests that the transplant site is a more permissive environment for growing axons, but also that the host tissue is not permissive enough for re-entry. Such results suggest that limiting the transplantation of stem cells into the lesion cavity may be less effective in promoting long-distance regeneration, than utilizing multiple transplant locations to create a continual bridge of permissive substrate (Brock et al., 2010). A short distance growth response of axons into a lesion site, in mid-thoracic SCI, will likely not re-connect with de-innervated lower motor neurons, interneurons driving central pattern generators, or sensory nuclei, which are responsible for producing locomotion and guiding movement. Although our study has shown that stem cells can be used to provide growth-stimulating proteins, such as SDF- $1 \alpha$, into an injured spinal cord, the need for guided, long-distance axon regeneration still remains.

Contrary to previous reports of MSC transplantations in SCI, this study failed to demonstrate a reduction in reactive gliosis, or inflammation, despite observing trends that are consistent with previous literature (Abrams et al., 2009). Studies examining the expression of key molecules, post-SCI, such as GFAP, CSPGs, or IBA-1, have suggested a return towards baseline from an elevated status around 30-days post-injury (Gwak, Kang, Unabia, \& Hulsebosch, 2012; Jones, Margolis, \& Tuszynski, 2003; Kumamaru et al., 2013; Lau et al., 2012). This stabilization of the injury environment at earlier time points, postinjury, may explain the inability to detect differences between groups at 7-weeks post-injury.

A concern that may arise when using any variant of SDF-1 in a treatment protocol is the role of SDF-1 in recruiting both local and systemic inflammation. However, as demonstrated by Zendedel and colleagues (2016), one of the functions of SDF- $1 \alpha$ during inflammation may be to selectively recruit either M2-activated microglia and/or CD4 ${ }^{+}$T-cells, both of which are thought to provide additional trophic support, as opposed to exerting cytotoxic effects. Despite the overexpression of SDF- $1 \alpha$ by MSCs in this study, no significant differences in the inflammatory marker, IBA-1, was found between groups. As MSCs have been known to exert moderate, anti-inflammatory effects, it may be possible that the anti-inflammatory cytokines produced by the MSCs partially attenuated any increase in inflammation that would have otherwise occurred by introducing SDF$1 \alpha$ alone (Rossignol et al., 2009).

Despite a lack of between-group differences on measures of white matter integrity at the lesion epicenter, the lesion environment in the SDF-1-MSC transplant group provided for significantly less cavitation compared to vehicle controls. Although the precise composition of the tissue existing within the lesion environment was not identified in this study, there was a robust density of CSPGs and IBA-1 labeled cells in the tissue within the lesion site. As CSPGs have been reported to lead to the collapse of the growth cone, the fact that the transplant/lesion site was dense in CSPGs, while also containing a greater labeling of GAP-43 in SDF-1-MSC-treated animals, creates a perplexing contradiction (Beller et al., 2013; Schmalfeldt, Bandtlow, Dours-Zimmermann, Winterhalter, \& Zimmermann, 2000). Such findings are suggestive that the secretome of MSCs, 
particularly when enhanced with SDF- $1 \alpha$ expression, can help overcome the inhibitory nature that CSPGs exert on growth and regeneration. Our findings help underscore the importance of maintaining tissue or replacing ECM within the lesion cavity so that growing axons can penetrate into the damaged area and encounter a permissive substrate for growth.

Although transplantation of SDF-1-MSCs reduced total cavitation in the present study, a considerable amount of cavitation remained in some rats, which could complicate the regrowth of axons into, or beyond, the lesion site in those rats with more severe injuries. The transplantation of MSCs, and especially SDF-1-MSCs, created a permissive environment for axonal growth. However, the inability to completely fill or reduce tissue loss in the lesion area suggests the need for either optimizing the dosage of the transplant, or utilizing live imaging techniques to ensure complete replacement of the ECM during transplantation. Addressing the concerns about restricting and filling the lesion cavity may be a critical first steps towards advancing regenerative techniques in clinically relevant contusion/compression animal models of SCI. In transection/hemisection models, the induced lesion can be easily filled and fused together with gel/stem-cell bridges, which have also shown to be a critical component for facilitating regeneration beyond the gliotic scar borders (Paul Lu et al., 2012). In contrast, contusion models, which are clinically relevant, and fraught with highly variable shapes and sizes of cavitation, and may require advanced imaging during transplant procedures to effectively fill the cavity.

\section{Conclusion}

In conclusion, this study has demonstrated that MSCs that have been genetically manipulated to overexpress SDF-1 $\alpha$, can enhance the secretion of paracrine agents, as evidenced by the enhanced chemotactic migration of NSCs in vitro. The accumulated in vitro and in vivo data obtained from this study provides a proof-of-principle that MSCs that overexpress SDF- $1 \alpha$ can reduce cavitation post-SCI, enhance the growth of axons into the transplant location, and improve functional performance of rats with moderately severe injuries. However, due to the tendencies of SDF- $1 \alpha$ to promote inflammation and metastasis of cancer cells (Luker et al., 2012) future work needs to be performed to evaluate long-term safety of this type of therapy. Similarly, future work is needed to identify the specific spinal tracts influenced by SDF- $1 \alpha$ expression, in order to better understand how this chemokine may be used as a therapy to enhance axon regeneration within the damaged spinal cord.

\section{Acknowledgments}

Support for this study was provided by the Office of Research and Sponsored Programs at CMU, the College of Medicine, the Jeff Lichon Spinal Cord Injury Foundation, the Field Neurosciences Institute and John G. Kulhavi Professorship.

\section{References}

Abrams, M.B., Dominguez, C., Pernold, K., Reger, R., WiesenfeldHallin, Z., Olson, L., \& Prockop, D. (2009). Multipotent mesenchymal stromal cells attenuate chronic inflammation and injury-induced sensitivity to mechanical stimuli in experimental spinal cord injury. Restorative Neurology and Neuroscience, 27(4), 307-321. https://doi.org/10.3233/RNN2009-0480

Alluin, O., Delivet-Mongrain, H., Gauthier, M.-K., Fehlings, M. G., Rossignol, S., \& Karimi-Abdolrezaee, S. (2014). Examination of the combined effects of chondroitinase $A B C$, growth factors and locomotor training following compressive spinal cord injury on neuroanatomical plasticity and kinematics. PLoS One, 9(10), e111072. https://doi.org/10.1371/journal. pone. 0111072

Alto, L.T., Havton, L.A., Conner, J.M., Hollis, E.R., Blesch, A., \& Tuszynski, M.H. (2009). Chemotropic guidance facilitates axonal regeneration and synapse formation after spinal cord injury. Nature Neuroscience, 12(9), 1106-1113. https://doi. org/10.1038/nn.2365

Basso, D.M., Beattie, M.S., \& Bresnahan, J.C. (1995). A sensitive and reliable locomotor rating scale for open field testing in rats. Journal of Neurotrauma, 12(1), 1-21. https://doi.org/ 10.1089/neu.1995.12.1

Beller, J.A., Kulengowski, B., Kobraei, E.M., Curinga, G., Calulot, C.M., Bahrami, A., . . . Snow, D.M. (2013). Comparison of sensory neuron growth cone and filopodial responses to structurally diverse aggrecan variants, in vitro. Experimental Neurology, 247, 143-157. https://doi.org/10.1016/j.expneurol. 2013.02.012

Bodea, G.O., Spille, J.-H., Abe, P., Andersson, A.S., Acker-Palmer, A., Stumm, R., ... Blaess, S. (2014). Reelin and CXCL12 regulate distinct migratory behaviors during the development of the dopaminergic system. Development (Cambridge, England), 141(3), 661-673. https://doi.org/10.1242/dev.099937

Brock, J.H., Rosenzweig, E.S., Blesch, A., Moseanko, R., Havton, L.A., Edgerton, V.R., \& Tuszynski, M.H. (2010). Local and remote growth factor effects after primate spinal cord injury. The Journal of Neuroscience: The Official Journal of the Society for Neuroscience, 30(29), 9728-9737. https://doi.org/10.1523/JNEUROSCI.1924-10.2010

Chalasani, S.H., Sabelko, K.A., Sunshine, M.J., Littman, D.R., \& Raper, J.A. (2003). A chemokine, SDF-1, reduces the 
effectiveness of multiple axonal repellents and is required for normal axon pathfinding. The Journal of Neuroscience: The Official Journal of the Society for Neuroscience, 23(4), 1360-1371.

Crane, A.T., Rossignol, J., \& Dunbar, G.L. (2014). Use of Genetically Altered Stem Cells for the Treatment of Huntington's Disease. Brain Sciences, 4(1), 202-219. https://doi.org/ 10.3390/brainsci4010202

Curtis, R., Green, D., Lindsay, R.M., \& Wilkin, G.P. (1993). Upregulation of GAP-43 and growth of axons in rat spinal cord after compression injury. Journal of Neurocytology, 22(1), 51-64.

Dey, N.D., Bombard, M.C., Roland, B.P., Davidson, S., Lu, M., Rossignol, J., . . Dunbar, G.L. (2010). Genetically engineered mesenchymal stem cells reduce behavioral deficits in the YAC 128 mouse model of Huntington's disease. Behavioural Brain Research, 214(2), 193-200. https://doi.org/10.1016/ j.bbr.2010.05.023

Dziembowska, M., Tham, T.N., Lau, P., Vitry, S., Lazarini, F., \& Dubois-Dalcq, M. (2005). A role for CXCR4 signaling in survival and migration of neural and oligodendrocyte precursors. Glia, 50(3), 258-269. https://doi.org/10.1002/glia.20170

Filippo, T.R.M., Galindo, L.T., Barnabe, G.F., Ariza, C.B., Mello, L.E., Juliano, M.A., ... Porcionatto, M.A. (2013). CXCL12 $\mathrm{N}$-terminal end is sufficient to induce chemotaxis and proliferation of neural stem/progenitor cells. Stem Cell Research, 11(2), 913-925. https://doi.org/10.1016/j.scr.2013.06.003

Gwak, Y.S., Kang, J., Unabia, G.C., \& Hulsebosch, C.E. (2012). Spatial and temporal activation of spinal glial cells: Role of gliopathy in central neuropathic pain following spinal cord injury in rats. Experimental Neurology, 234(2), 362-372. https://doi.org/10.1016/j.expneurol.2011.10.010

Hoffman, P.N. (1989). Expression of GAP-43, a rapidly transported growth-associated protein, and class II beta tubulin, a slowly transported cytoskeletal protein, are coordinated in regenerating neurons. The Journal of Neuroscience: The Official Journal of the Society for Neuroscience, 9(3), 893-897.

Jones, L.L., Margolis, R.U., \& Tuszynski, M.H. (2003). The chondroitin sulfate proteoglycans neurocan, brevican, phosphacan, and versican are differentially regulated following spinal cord injury. Experimental Neurology, 182(2), 399-411.

Kadoya, K., Tsukada, S., Lu, P., Coppola, G., Geschwind, D., Filbin, M.T., ... Tuszynski, M.H. (2009). Combined intrinsic and extrinsic neuronal mechanisms facilitate bridging axonal regeneration one year after spinal cord injury. Neuron, 64(2), 165-172. https://doi.org/10.1016/j.neuron.2009.09.016

Kozak, M. (1987). An analysis of 5'-noncoding sequences from 699 vertebrate messenger RNAs. Nucleic Acids Research, $15(20), 8125-8148$.

Kumagai, G., Tsoulfas, P., Toh, S., McNiece, I., Bramlett, H.M., \& Dietrich, W.D. (2013). Genetically modified mesenchymal stem cells (MSCs) promote axonal regeneration and prevent hypersensitivity after spinal cord injury. Experimental Neurology, 248, 369-380. https://doi.org/10.1016/j.expneurol. 2013.06.028

Kumamaru, H., Saiwai, H., Kubota, K., Kobayakawa, K., Yokota, K., Ohkawa, Y., ... Okada, S. (2013). Therapeutic activities of engrafted neural stem/precursor cells are not dormant in the chronically injured spinal cord. Stem Cells (Dayton, Ohio), 31(8), 1535-1547. https://doi.org/10.1002/stem.1404
Lau, L.W., Keough, M.B., Haylock-Jacobs, S., Cua, R., Döring, A., Sloka, S., ... Yong, V.W. (2012). Chondroitin sulfate proteoglycans in demyelinated lesions impair remyelination. Annals of Neurology, 72(3), 419-432. https://doi.org/10.1002/ ana.23599

Li, J.Y., Kling-Petersen, A., \& Dahlström, A. (1993). GAP 43like immunoreactivity in normal adult rat sciatic nerve, spinal cord, and motoneurons: Axonal transport and effect of spinal cord transection. Neuroscience, 57(3), 759-776.

Li, M., \& Ransohoff, R.M. (2008). Multiple roles of chemokine CXCL12 in the central nervous system: A migration from immunology to neurobiology. Progress in Neurobiology, 84(2), 116-131. https://doi.org/10.1016/j.pneurobio.2007.11. 003

Li, X.-Y., Ding, J., Zheng, Z.-H., Li, X.-Y., Wu, Z.-B., \& Zhu, P. (2012). Long-term culture in vitro impairs the immunosuppressive activity of mesenchymal stem cells on T cells. Molecular Medicine Reports, 6(5), 1183-1189. https:// doi.org/10.3892/mmr.2012.1039

Lieberam, I., Agalliu, D., Nagasawa, T., Ericson, J., \& Jessell, T.M. (2005). A Cxcl12-CXCR4 chemokine signaling pathway defines the initial trajectory of mammalian motor axons. Neuron, 47(5), 667-679. https://doi.org/10.1016/j.neuron.2005. 08.011

Lu, P., Blesch, A., Graham, L., Wang, Y., Samara, R., Banos, K., ... Tuszynski, M.H. (2012). Motor axonal regeneration after partial and complete spinal cord transection. The Journal of Neuroscience: The Official Journal of the Society for Neuroscience, 32(24), 8208-8218. https://doi.org/10.1523/JNEUR OSCI.0308-12.2012

Lu, P., Jones, L.L., \& Tuszynski, M.H. (2005). BDNF-expressing marrow stromal cells support extensive axonal growth at sites of spinal cord injury. Experimental Neurology, 191(2), 344360. https://doi.org/10.1016/j.expneurol.2004.09.018

Lu, P., Yang, H., Jones, L.L., Filbin, M.T., \& Tuszynski, M.H. (2004). Combinatorial therapy with neurotrophins and cAMP promotes axonal regeneration beyond sites of spinal cord injury. The Journal of Neuroscience: The Official Journal of the Society for Neuroscience, 24(28), 6402-6409. https:// doi.org/10.1523/JNEUROSCI.1492-04.2004

Luker, K.E., Lewin, S.A., Mihalko, L.A., Schmidt, B.T., Winkler, J.S., Coggins, N.L., ... Luker, G.D. (2012). Scavenging of CXCL12 by CXCR7 promotes tumor growth and metastasis of CXCR4-positive breast cancer cells. Oncogene, 31(45), 4750-4758. https://doi.org/10.1038/onc.2011.633

Metz, G.A., Curt, A., van de Meent, H., Klusman, I., Schwab, M. E., \& Dietz, V. (2000). Validation of the weight-drop contusion model in rats: A comparative study of human spinal cord injury. Journal of Neurotrauma, 17(1), 1-17. https://doi.org/ 10.1089/neu.2000.17.1

Neuhuber, B., Timothy Himes, B., Shumsky, J.S., Gallo, G., \& Fischer, I. (2005). Axon growth and recovery of function supported by human bone marrow stromal cells in the injured spinal cord exhibit donor variations. Brain Research, 1035(1), 73-85. https://doi.org/10.1016/j.brainres.2004.11.055

Oliveri, R.S., Bello, S., \& Biering-Sørensen, F. (2014). Mesenchymal stem cells improve locomotor recovery in traumatic spinal cord injury: Systematic review with meta-analyses of rat models. Neurobiology of Disease, 62, 338-353. https:// doi.org/10.1016/j.nbd.2013.10.014 
Orimo, A., Gupta, P.B., Sgroi, D.C., Arenzana-Seisdedos, F., Delaunay, T., Naeem, R., ... Weinberg, R.A. (2005). Stromal fibroblasts present in invasive human breast carcinomas promote tumor growth and angiogenesis through elevated SDF-1/CXCL12 secretion. Cell, 121(3), 335-348. https://doi. org/10.1016/j.cell.2005.02.034

Rossignol, J., Boyer, C., Thinard, R., Remy, S., Dugast, A.-S., Dubayle, D., ... Lescaudron, L. (2009). Mesenchymal stem cells induce a weak immune response in the rat striatum after allo or xenotransplantation. Journal of Cellular and Molecular Medicine, 13(8B), 2547-2558. https://doi.org/10.1111/ j.1582-4934.2009.00657.x

Rossignol, J., Fink, K.D., Crane, A.T., Davis, K.K., Bombard, M. C., Clerc, S., ...Dunbar, G.L. (2015). Reductions in behavioral deficits and neuropathology in the R6/2 mouse model of Huntington's disease following transplantation of bone-marrow-derived mesenchymal stem cells is dependent on passage number. Stem Cell Research \& Therapy, 6, 9. https://doi.org/10.1186/scrt545

Ruitenberg, M.J., Levison, D.B., Lee, S.V., Verhaagen, J., Harvey, A.R., \& Plant, G.W. (2005). NT-3 expression from engineered olfactory ensheathing glia promotes spinal sparing and regeneration. Brain: A Journal of Neurology, 128(Pt 4), 839-853. https://doi.org/10.1093/brain/awh424

Sasaki, M., Radtke, C., Tan, A.M., Zhao, P., Hamada, H., Houkin, K., ... Kocsis, J.D. (2009). BDNF-hypersecreting human mesenchymal stem cells promote functional recovery, axonal sprouting, and protection of corticospinal neurons after spinal cord injury. The Journal of Neuroscience: The Official Journal of the Society for Neuroscience, 29(47), 14932-14941. https://doi.org/10.1523/JNEUROSCI.2769-09.2009

Schmalfeldt, M., Bandtlow, C.E., Dours-Zimmermann, M.T., Winterhalter, K.H., \& Zimmermann, D.R. (2000). Brain derived versican V2 is a potent inhibitor of axonal growth. Journal of Cell Science, 113 (Pt 5), 807-816.

Shang, A.-J., Hong, S.-Q., Xu, Q., Wang, H.-Y., Yang, Y., Wang, Z.-F., .. Xu, R.-X. (2011). NT-3-secreting human umbilical cord mesenchymal stromal cell transplantation for the treatment of acute spinal cord injury in rats. Brain Research, 1391, 102-113. https://doi.org/10.1016/j.brainres.2011.03.019

Somers, M. (2000). Spinal Cord Injury: Functional Rehabilitation (2nd Edition). Prentice Hall.

Song, Q., Xu, R., Zhang, Q., Ma, M., \& Zhao, X. (2014). Therapeutic effect of transplanting bone mesenchymal stem cells on the hind limbs' motor function of rats with acute spinal cord injury. International Journal of Clinical and Experimental Medicine, 7(1), 262-267.
Taylor, L., Jones, L., Tuszynski, M.H., \& Blesch, A. (2006). Neurotrophin- 3 gradients established by lentiviral gene delivery promote short-distance axonal bridging beyond cellular grafts in the injured spinal cord. The Journal of Neuroscience: The Official Journal of the Society for Neuroscience, 26(38), 9713-9721. https://doi.org/10.1523/JNEUROSCI.07 34-06.2006

Toba, Y., Tiong, J.D., Ma, Q., \& Wray, S. (2008). CXCR4/SDF-1 system modulates development of GnRH-1 neurons and the olfactory system. Developmental Neurobiology, 68(4), 487503. https://doi.org/10.1002/dneu.20594

Tuszynski, M.H., \& Steward, O. (2012). Concepts and methods for the study of axonal regeneration in the CNS. Neuron, 74(5), 777-791. https://doi.org/10.1016/j.neuron.2012.05.006

Xue, L., Wang, J., Wang, W., Yang, Z., Hu, Z., Hu, M., \& Ding, P. (2014). The effect of stromal cell-derived factor 1 in the migration of neural stem cells. Cell Biochemistry and Biophysics, 70(3), 1609-1616. https://doi.org/10.1007/s12013014-0103-5

Yasuda, A., Tsuji, O., Shibata, S., Nori, S., Takano, M., Kobayashi, Y., ... Okano, H. (2011). Significance of remyelination by neural stem/progenitor cells transplanted into the injured spinal cord. Stem Cells (Dayton, Ohio), 29(12), 1983-1994. https://doi.org/10.1002/stem.767

Zendedel, A., Johann, S., Mehrabi, S., Joghataei, M.-T., Hassanzadeh, G., Kipp, M., \& Beyer, C. (2016). Activation and regulation of NLRP3 inflammasome by intrathecal application of SDF-1a in a spinal cord injury model. Molecular Neurobiology, 53(5), 3063-3075. https://doi.org/10.1007/s12035-0159203-5

Zendedel, A., Nobakht, M., Bakhtiyari, M., Beyer, C., Kipp, M., Baazm, M., \& Joghataie, M.T. (2012). Stromal cellderived factor-1 alpha (SDF-1 $\alpha$ ) improves neural recovery after spinal cord contusion in rats. Brain Research, 1473, 214-226. https://doi.org/10.1016/j.brainres.2012.07.037

Zhang, Y.-J., Zhang, W., Lin, C.-G., Ding, Y., Huang, S.-F., Wu, J.-L., ... Zeng, Y.-S. (2012). Neurotrophin-3 gene modified mesenchymal stem cells promote remyelination and functional recovery in the demyelinated spinal cord of rats. Journal of the Neurological Sciences, 313(1-2), 64-74. https://doi.org/10.1016/j.jns.2011.09.027

Zhu, Y., Matsumoto, T., Mikami, S., Nagasawa, T., \& Murakami, F. (2009). SDF1/CXCR4 signalling regulates two distinct processes of precerebellar neuronal migration and its depletion leads to abnormal pontine nuclei formation. Development (Cambridge, England), 136(11), 1919-1928. https://doi.org/ 10.1242/dev.032276 